\title{
Provider-initiated HIV testing and counseling in Mbeya City, south-western Tanzania: knowledge and practice of health care providers
}

\author{
NTULI KAPOLOGWE ${ }^{1 *}$, MICHAEL MAHANDE ${ }^{2}$ and SIA E. MSUYA ${ }^{2}$ \\ Department of Health, Bahi District Council, P.O Box 2993, Dodoma, Tanzania \\ KCM College of Tumaini University, Community Health Department, P.O Box 2240, Moshi, Tanzania
}

\begin{abstract}
Provider-initiated testing and counseling (PITC) is a routine HIV counseling and testing offered to adult clients attending at health care facilities. In Tanzania PITC started in 2007, with the aim of increasing early HIV diagnosis and timely access to care, treatment and support services. The objective of this study was to assess the level of knowledge and practice of health care providers on PITC. This cross sectional study was conducted in April-May 2010 among nurses and clinicians working in the government health care facilities in Mbeya City, south-western Tanzania. Self-administered questionnaire was used to obtain relevant information from the study participants. A total of 402 ( $95 \%$ response rate) care providers were interviewed. Their mean $( \pm \mathrm{SD})$ age was $41 \pm 9.5$ years. The majority $304(76 \%)$ were nurses. Their duration of practice ranged from 4 months to 39 years. All the care providers reported to be aware of PITC services. However, 35\% of them had inadequate knowledge of PITC and $269(67 \%)$ reported to have ever offered PITC services. Median number of clients attended per week was 3 (IQR, 1-6 clients). Participants who had attended special training on PITC were 6-fold more likely to offer PITC services $[\mathrm{OR}=6.2,95 \% \mathrm{CI}=3.7-10.2 ; P<0.001]$ than those who had not attended. In conclusion more than one-third of health providers do not routinely offer PITC in Mbeya City, leading to missed opportunity for early HIV diagnosis. On job and in-service training to improve PITC knowledge and supportive supervision for those trained is required.
\end{abstract}

Keywords: Knowledge, practice, provider-initiated testing and counseling, Tanzania

\section{Introduction}

Human Immunodeficiency Virus (HIV) and Acquired Immunodeficiency Syndrome (AIDS) is a global health threat and continue to pose great public health challenges. It is estimated that, worldwide about 33.4 million people are living with HIV (UNAIDS, 2009). In 2008, approximately 2.7 million people were infected with HIV and 2 million died from AIDS (UNAIDS, 2009). Sub-Saharan Africa has been hit hard by the pandemic and it is currently estimated to have $70 \%$ of people living with HIV/AIDS worldwide (UNAIDS, 2009). HIV is a public health problem in Tanzania as well, with a prevalence of 5.7\% among adults aged 15-49 years. However, the prevalence varies substantially between regions and districts throughout the country (THMIS, 2008).

Previous studies have reported that only $10-12 \%$ of people in sub-Saharan Africa know their HIV status (UNAIDS, 2009). Although there are some efforts to promote and support HIV counseling and testing services in Tanzania, the majority (85\%) of the populations are unaware of their HIV status (THMIS, 2008). Since 2007, UNAIDS and WHO have recommended that countries with generalized HIV epidemics should adopt a policy of Provider Initiated Testing and Counseling (PITC) in clinical settings. The policy guidelines suggest that HIV testing and

${ }^{*}$ Correspondence: Ntuli Kapologwe; Email: nkapologwe2002@gmail.com 
counseling should be offered by the health care provider as part of the routine care to all patients or clients attending at health facilities, regardless of whether or not the patient shows signs and symptoms of underlying HIV infection (UNAIDS/WHO, 2007).

PITC is an active case finding strategy, and relies on health care providers working at clinical settings, to routinely offer HIV counseling, diagnostic HIV testing, and HIV screening. It is also emphasized that PITC should be voluntary and that the "Three C's" - informed consent, counseling and confidentiality must be observed. The PITC policy complements the existing HIV voluntary counseling and testing (VCT) programmes which rely on individuals to selfrefer for testing (THMIS, 2008). With these two strategies PITC and VCT, it is hoped that the number of people knowing their HIV-status will increase, and people will be detected earlier, in order to access care and treatment in timely manner (Kiene et al., 2010).

In Mbeya Region, the implementation of the PITC programme started in 2007. This was preceded by training of health care providers in PITC which was facilitated by the Ministry of Health and Social Welfare in collaboration with non-governmental organizations. There is however limited information on health care providers' knowledge, practice as well as barriers to PITC provision since the scale up in 2007. This study therefore, aimed to assess PITC knowledge and practice of health care providers working in the government health facilities in Mbeya City in south-western Tanzania.

\section{Materials and Methods}

\section{Study area and sampling procedures}

A cross-sectional study was conducted from April to May 2010 among nurses and clinicians working in government health facilities of Mbeya City, south-western, Tanzania. The City has a population of 233,441 lives (National Bureau of Statistics, 2009) and HIV prevalence of $12.5 \%$ (THMIS, 2008). There were 12 government health facilities in the city at the time of the study. These included one referral hospital, one regional hospital and ten primary health care (PHC) facilities which include dispensaries and health centre.

Study participants were clinicians (medical doctors, assistant medical doctors and clinical officers) and nurses working in the health facilities. A list of all clinicians and nurses was obtained from the Regional Medical Officer's office. A stratified random sampling method with a probability proportional to size of the population was used to select the participants. The proportion of providers selected from each level was calculated based on their number. At the time of the study, 53\% of all clinicians and nurses working in Mbeya Referral Hospital, 35\% in PHCs and 12\% in Mbeya Regional Hospital. At each facility, the list of providers was stratified according to cadre (i.e. nurses or clinicians). This was necessary because the clinician: nurse ratio was 1:3. Simple random sampling was then used to select the participants from a sampling frame which had the names of providers at each level. A total of 54 clinicians and 160 nurses were selected in Mbeya Referral Hospital, 31 clinicians and 109 nurses were selected from PHC, and 13 clinicians and 35 nurses respectively were selected from Mbeya Regional Hospital. 


\section{Data collection}

A standardized questionnaire was used to collect information on demographic characteristics, cadre, previous training on HIV counseling and testing, training on PITC, number of years at service and on knowledge and practice regarding PITC. Information regarding knowledge and practice were assessed using dichotomous type of questions. A total of 16 questions were used to assess the PITC knowledge. Of the 16, two questions which focused on basic principles of PITC were given a score of 3 points for a correct response and the rest were given a score of one point for each correct answer, making a total score of 20 points. In order for a participant to have adequate knowledge, one had to get correct responses from two questions focused on the basic principles of PITC and at least nine points from other questions. The participants scoring 15 or more points were categorized to have adequate PITC knowledge. The cut off points was based on previous research (USAID-Tanzania, 2007).

Practice of PITC was measured by first asking the participants if they had ever offered PITC. Those who had "ever offered PITC" were further categorized into "active" and "inactive" PITC providers based on their performance in the past one month of practice. Health care providers who had not offered PITC service to any patient or client during the past month of their practice were classified as "inactive" while those who offered it to at least one patient or clients were classified as "active". Providers who have ever offered PITC were further asked 12 questions to assess if they adhered to the three PITC principles of confidentiality, counselling and informed consent. Counselling domain consisted of 3 questions, confidentiality 5 questions and informed consent 4 questions. The cut-off was set at 10 points; those who scored 10 or more points were regarded as offering good PITC service whereas those who scored less than 10 points were considered to be offering poor PITC service.

\section{Data analysis}

Data were entered and analyzed using Statistical Package for Social Science (SPSS) programme version 17.0 (SPSS Inc., Chicago, IL, USA). Bivariate analysis was carried out to assess the association of PITC knowledge and practice with predictor values. Multiple logistic regression analysis was carried out to control for confounders and get independent predictors of PITC practice and knowledge. Only variables shown statistical significance in the bivariate analysis at $(\mathrm{p} \leq 0.05)$ were entered in the regression model.

\section{Ethical considerations}

Ethical approval was obtained from KCM-College Research Ethics Committee prior the commencement of the study. Permission to conduct the study was sought from the local administrative authority. The informed consent was obtained from all participants after being explained of the aim of the study. For confidentiality and privacy, identification numbers were used instead of names.

\section{Results}

A total of 402 (95\% response rate) participants were involved in the study. The majority were aged $\leq 35$ years (mean age $=41 \pm 9.5$ ) years. About two thirds $(64.7 \%)$ of the participants were 
females and $78.9 \%$ were ever married. Those with lower qualifications (298) accounted for the majority of the health care providers. Their years of practice ranged from 4 months to 39 years with median of 9 years [(IQR), 4-20 years] where, majority $(75.6 \%)$ practiced for less than 10 years (Table 1).

Table 1: Association between practices of PITC by health care provider with socio-demographic characteristics, training, knowledge on PITC $(n=402)$

\begin{tabular}{|c|c|c|c|c|c|}
\hline \multirow[t]{3}{*}{ Variable } & \multirow[t]{3}{*}{ Response } & \multirow[t]{3}{*}{ Total } & \multicolumn{2}{|c|}{ Practice of PITC } & \multirow[t]{3}{*}{$P$-value } \\
\hline & & & Ever offered & $\begin{array}{l}\text { Never } \\
\text { offered }\end{array}$ & \\
\hline & & & No. $(\%)$ & No. $(\%)$ & \\
\hline \multirow[t]{2}{*}{ Sex } & Female & 260 & $177(68.8)$ & $83(31.9)$ & \\
\hline & Male & 142 & $92(64.8)$ & $50(35.2)$ & 0.503 \\
\hline \multirow[t]{2}{*}{ Age (years } & Younger age ( $\leq 35$ years) & 303 & 187 (61.7) & $116(38.3)$ & \\
\hline & Older age ( $\geq 36$ years) & 99 & $82(82.8)$ & $17(17.2)$ & $<0.001$ \\
\hline \multirow[t]{2}{*}{ Marital status } & Never married & 85 & $63(74.1)$ & $22(25.9)$ & \\
\hline & Ever married & 317 & $206(65.0)$ & $111(35.0)$ & 0.112 \\
\hline \multirow[t]{2}{*}{ Highest qualification } & Lower qualification & 298 & $196(65.8)$ & $102(34.2)$ & \\
\hline & Higher qualification & 104 & $73(70.2)$ & $31(29.8)$ & 0.409 \\
\hline \multirow[t]{2}{*}{ Profession } & Nurse & 304 & $206(67.8)$ & $98(32.2)$ & \\
\hline & Clinician & 98 & $63(64.3)$ & $35(35.7)$ & 0.525 \\
\hline \multirow[t]{2}{*}{ Years of practice } & Short ( $\leq 10$ years) & 237 & $141(59.5)$ & $96(40.5)$ & \\
\hline & Long ( $\geq 11$ years) & 165 & $128(77.6)$ & $37(22.4)$ & $<0.001$ \\
\hline \multirow[t]{2}{*}{ Training status on PITC } & Attended & 215 & $184(85.6)$ & $31(14.4)$ & \\
\hline & Not attended & 187 & $85(45.5)$ & $102(54.5)$ & $<0.001$ \\
\hline \multirow[t]{2}{*}{ Knowledge on PITC } & Adequate & 260 & 187 (71.9) & $73(28.1)$ & \\
\hline & Inadequate & 142 & $82(57.7)$ & $60(42.3)$ & 0.004 \\
\hline \multirow[t]{2}{*}{ Type of facility } & Dispensary/Health centre & 140 & $83(59.3)$ & $57(40.7)$ & \\
\hline & Regional/Referral hospital & 262 & $186(71.0)$ & $76(29.0)$ & 0.017 \\
\hline \multirow[t]{2}{*}{ Department/Unit } & Out-patient department & 278 & $184(66.2)$ & $94(33.8)$ & \\
\hline & In- patient department & 124 & $85(68.5)$ & $39(31.5)$ & 0.642 \\
\hline
\end{tabular}

All the 402 health care providers reported to have heard about PITC. The common reported sources of information on PITC were professional colleagues (66.4\%), training on PITC (53.5\%) and conferences (23.9\%). Sixty five percent (260) of the participants had adequate knowledge on PITC. Fifty-three percent (215) had attended special training on PITC, but there was a variation in the duration of training with majority $(70.7 \%)$ reported to have had a one-week training, $25.1 \%$ two-week and $4.2 \%$ three-weeks or more of training. Knowledge of health care providers on PITC was significantly associated with PITC training $(P \leq 0.001)$. Participants who attended training on PITC irrespective of their duration of training demonstrated an increase of their PITC knowledge $(75 \%)$ compared to others $(22 \%)$. In addition, participants who were ever married had three-folds adequate knowledge on PITC [OR=2.6, 95\% CI $=1.6-4.3$ ] compared to 
their counterpart who never married. About $67 \%(n=269)$ of participants reported to have ever offered PITC services. Majority $(78.1 \%)$ of the participants were actively involved in provision of PITC service. The self-reported number of patients attended by each participant in the past one month prior to the interview varied from 0 to 55 with a median number of 3 clients (IQR, 16 clients).

Old age, long duration of practice, attendance of PITC training and adequate knowledge on PITC were all significantly associated with ever offering PITC service $(P=<0.001)$. Older health care providers were three folds more likely to offer PITC services [OR $=2.9,95 \% \mathrm{CI}=1.7$ - 5.3], compared to their young counterpart. Additionally, health care providers who had practiced for more than ten years were two times more likely to have had offered PITC than those who practiced less than 10 years. [OR $=2.4,95 \% \mathrm{CI}=1.5-3.7]$. Having attended PITC training $[\mathrm{OR}=7.1,95 \% \mathrm{CI}=4.4-11.5]$, and having adequate knowledge of PITC $[\mathrm{OR}=1.9,95 \%$ $\mathrm{CI}=1.2-2.9]$ were both significantly associated with offering PITC services. Logistic regression analysis revealed that only two variables were independent predictors of offering PITC services (Table 2). Special training on PITC $[\mathrm{OR}=6.2,95 \% \mathrm{CI}=3.7-10.2]$, as well as working at regional/referral hospital $[\mathrm{OR}=1.9,95 \% \mathrm{CI}=1.1-3.0]$ were significantly associated with offering PITC services $(P \leq 0.001)$. Most of the socio-demographic variables were not associated with offering good PITC services after adjustment.

Table 2: Logistic regression model of Practice of PITC by health care providers' characteristics

\begin{tabular}{|c|c|c|c|c|}
\hline Predictor variable & Response & OR $^{*}$ & $95 \%$ CI & P-value \\
\hline \multirow[t]{2}{*}{ Knowledge on PITC } & Adequate & 1.2 & $0.8-2.1$ & 0.382 \\
\hline & Inadequate & 1 & & \\
\hline \multirow[t]{2}{*}{ Years of practice } & Long in-service & 1.6 & $0.9-2.9$ & 0.092 \\
\hline & Short in-service & 1 & & \\
\hline \multirow[t]{2}{*}{ Special training on PITC } & Attended & 6.2 & $3.7-10.2$ & $<0.001$ \\
\hline & Not attended & 1 & & \\
\hline \multirow[t]{2}{*}{ Type of health facility } & Regional/Referral Hospital & 1.9 & $1.1-3.0$ & 0.014 \\
\hline & Dispensary/ Health Centre & 1 & & \\
\hline \multirow[t]{2}{*}{ Age group } & Older age & 1.7 & $0.8-3.5$ & 0.137 \\
\hline & Young age & 1 & & \\
\hline
\end{tabular}

*OR = Adjusted Odds Ratio; $\mathbf{C I}=$ Confidence Interval

Of the 269 health care providers who reported to have ever offered PITC service, $(70.6 \% ; \mathrm{N}=$ 190) were offering good PITC services and other offered poor practice. On the other hand, those who had practiced for short duration ( $\leq 10$ years) were found to be offering good PITC services than those who have been in practice for more than 10 years $(P=0.012)$. In addition, participants who attended special PITC training (75\%) were two fold more likely to offer good PITC services compared to their counterparts (61\%) have not had PITC training [OR $=1.9,95 \% \mathrm{CI}=1.1-3.4]$. Health care providers with adequate PITC knowledge were three times more likely to offer good PITC services compared to their counterpart $[\mathrm{OR}=3.3,95 \% \mathrm{CI}=1.2-5.8]$.

\section{Discussion}


The health care workers are the cornerstone for implementation of PITC strategy as required by the Tanzania Ministry of Health and Social welfare and the World Health Organization. In this study, all the participants were aware of PITC services. However, about one-third of health care providers was found to have inadequate knowledge on PITC. This figure is lower compared to that which was reported in a New York City study, in which two-thirds of the primary health care providers had no knowledge of the existence guidelines and approaches to PITC (Jain et al., 2009). The higher HIV prevalence in Mbeya Region compared to that of New York City might have contributed to the higher awareness and knowledge in Mbeya. Adequate PITC knowledge was associated with PITC provision in this study, and this highlights the importance of making sure that all the health care providers receive PITC training in this high HIV prevalence setting.

Marital status was associated with PITC knowledge. The health care providers who ever married were significantly found to have better knowledge on PITC compared to those who have never married. It is possible that, the ever married candidates might be those who have practiced for many years and therefore had adequate knowledge.

Special training on PITC was significantly associated with the PITC knowledge. In this study, the majority of health care providers who attended special training had adequate knowledge compared to those without training. Both knowledge and training were independently associated with provision of good PITC services. Similar findings have been reported in the USA and in Uganda (Burr et al., 2006; Wanyenze et al., 2006). The study in Uganda indicated that a significant proportion of health providers improve PITC practice after training. However, in our study about a quarter of those who attended special PITC training had inadequate knowledge. We observed a tendency of health care providers to forget what they were previously taught and problem might be with quality and duration in their previous training on PITC. The majority of health providers in this study received a one week training contrary to the 3 weeks of training on PITC as recommended by the World Health Organization (WHO, 2004). The short duration of study is likely to be due to financial constraints (MoHSW, 2007).

In this study, two-thirds of health care providers were reported to have ever offered PITC service and the over three quarters were actively providing the service. This proportion is higher than that reported in USA (20.3\%) and Canada (55\%) (Guenter et al., 2003; Cohan et al., 2009). Since the effective provision of PITC call for motivated health care providers who have desire to meet the client needs, it is likely that, health workers in the present study had higher motivation compared to their counterpart in the USA and Canada. Moreover, this study noted that significant proportions of health care providers were not actively involved in the provision of PITC. Our findings indicate that only about half of the providers are routinely offering PITC in this setting at any given time. This is a worrying trend as it highlights a missed opportunity for provision of PITC and thus early detection of HIV infections. Health managers in the area will need to investigate the reason for this pattern and come with solutions to improve PITC service provision (THMIS, 2008; MoHSW, 2007).

Health care providers working at regional and referral hospitals were two-fold more likely to offer PITC compared to those in primary health care facilities. This might be due to the fact that those who are working at the primary health care levels are overwhelmed with a lot of 
patients. The increased number of patients at the primary health care is most probably because it is the first gateway to the access of health care services in the health care system ladder compared to the higher facilities where they mainly receive referred patients. It is important therefore that human resource for capacity is strengthened to cope with the increasing need for HIV care and treatment services close to the community.

Training on PITC was significantly associated with offering good quality PITC services. This is supported by the previous study by Weaver et al., 2008. It is likely that the current training of PITC emphasized more on the relationship between patients and health care provider. This has beneficial effect on the patient satisfaction and hence increases in PITC uptake.

Despite of the importance of the information generated by this study, there some limitations which hinders the generalization of our findings to other settings. The study was cross-sectional in nature and relied on self-reported practices, both of which limits ability to draw conclusions about causal-effect relationships between health care providers' characteristics and PITC practice. In conclusion, most health providers had adequate knowledge on PITC and two thirds had ever provided PITC service. Level of knowledge and previous training in PITC had an influence in provision of PITC. However, although PITC is an effective strategy for early identification of unrecognized HIV infections, there is still missed opportunity to offer the PITC service that occurs at health facilities, as nearly a third of health care providers had never offered PITC and one fifth are not actively offering the service. Good quality training on PITC and take into account the recommended duration should be provided to all health care providers in this setting. The role of good quality training on PITC to all health care providers should not be undermined. The information in this study is potentially important for those who wish to scale-up the PITC, since it has shown various parameters associated with PITC practice.

\section{Acknowledgements}

The authors wish to acknowledge the respective health care workers, the District Medical Officer, Medical Officers' In-charge of all health facilities which participated in this study. Mbeya City Council Director is thanked for the support and cooperation rendered. Special thanks are to Julieth Kabengula, Imani Mwasomola and Samwel Lazaro for their assistance during data collection. This study received financial support from the Tanzania Ministry of Health and Social Welfare.

\section{References}

Burr, C.K., Storm, D.S. \& Gross, E. (2006) A faculty trainer model: increasing knowledge and changing practice to improve perinatal HIV prevention and care. AIDS Patient Care STDs 20, 183-192.

Cohan, D., Gomez, E., Dowling, T., Zelota, N., Kaplan, B. \& Klausner, J.D. (2009). HIV testing attitudes and practices among clinicians in the era of updated Centers for Disease 
Control and Prevention recommendations. Journal of Acquired Immune Deficiency Syndrome 50, 114-116.

Guenter, D., Kaczorowski, J., Carroll, J. \& Sellors, J. (2003) Prenatal HIV tests. Routine testing or informed choice? Canadian Family Physician 49, 1334-1340.

Jain, C.L., Wyatt, C.M., Burke, R., Sepkowitz, K. \& Begier, E.M. (2009) Knowledge of the Centers for Disease Control and prevention's 2006 routine HIV testing recommendations among New York City internal medicine residents. AIDS Patient Care STDs 23, 167-176.

Kiene, S.M., Bateganya, M., Wanyenze, R., Lule, H., Nantaba, H. \& Stein, M.D. (2010) Initial outcomes of provider-initiated routine HIV testing and counseling during outpatient care at a rural Ugandan hospital: risky sexual behavior, partner HIV testing, disclosure, and HIV care seeking. AIDS Patient Care STDs 24, 117-126.

NBS (2009) National Bureau of Statistics, Dar es Salaam, Tanzania.

Montano, D.E., Philips, W.R., Kasprzyk, D., Greek, A. (2008). STD/HIV prevention practices among primary care clinicians: risk assessment, prevention counseling, and testing. Sexually Transmitted Disease 35, 154-166.

MoHSW (2007) National Guidelines on Provider initiated Testing and Counseling. Ministry of Health and Social Welfare, United Republic of Tanzania. Available at: http://www.moh.go.tz/documents/ (Accessed on October 30th 2009).

MoHSW (2008) Guidelines for HIV Testing and Counseling in Clinical Setting. Ministry of Health and Social Welfare, United Republic of Tanzania. Available at: http://www.who.int/hiv/topics/vct/TZ_PITC-Guidelines_final edit_July2007.pdf.

THMIS (2008) Tanzania HIV/AIDS and Malaria Indicator Survey (THMIS) 2007/08. National Bureau of Statistics. Available at: http://www.nbs.go.tz/THIS/THIS2007-08/THIS200708.htm. (Accessed on 1st November 2009).

USAID-Tanzania (2007) Evaluation of Knowledge, Attitude and Practice of health care providers towards HIV-positive patients in Tanzania.

UNAIDS/WHO (2007) Guidance on Provider-Initiated Testing and Counseling in Health Facilities. World Health Organization/UNAIDS, Geneva.

UNAIDS (2009) Report on the Global HIV/AIDS epidemic. UNAIDS, Geneva.

Wanyenze, R., Kamya, M., Liechty, C.A., Ronald, A., Guzman, D.J., Wabwire-Mangen F., Mayanja-Kizza, H. \& Bangsberg DR. (2006) HIV Counseling and testing practices at an urban hospital in Kampala, Uganda. AIDS and Behavior 10, 361-367.

Weaver, M.R., Myaya, M., Disasi, K., Regoeng, M., Matumo, H.N., Madisa, M., Puttkammer, N., Speilberg, F., Kilmarx, P.H. \& Marrazzo, J.M. (2008) Routine HIV testing in the context of syndromic management of sexually transmitted infections: outcomes of the first phase of a training programme in Botswana. Sexually Transmitted Infections 84, 259-264.

WHO (2004) Rapid HIV Tests: Guidelines for Use in HIV Testing and Counseling Services in ResourceConstrained Settings. Geneva: WHO. 